\title{
Network pharmacology-based identification of potential targets of Triterpenoids from Liquidambaris Fructus on the treatment of Rheumatoid Arthritis
}

\section{Wenxuan Li}

China Medical University

\section{Ping Qian}

China Medical University

Li Gu

China Medical University

Dongfang Zhang ( $\square$ dfzhang@cmu.edu.cn)

China Medical University https://orcid.org/0000-0003-4785-7039

\section{Research}

Keywords: Rheumatoid arthritis, Liquidambaris Fructus, Triterpenoids, pharmacophore matching, enrichment analysis, molecular docking

Posted Date: May 23rd, 2020

DOI: https://doi.org/10.21203/rs.3.rs-29057/v1

License: (c) (1) This work is licensed under a Creative Commons Attribution 4.0 International License. Read Full License 


\section{Abstract \\ Background}

Rheumatoid arthritis (RA) is a chronic inflammatory autoimmune disease, and its exact etiology and pathogenesis are still unclear by now, therefore it is urgent to develop new therapeutic targets for RA treatment. Liquidambaris Fructus (LF) is used for joint pain, numbness and edema, and triterpenoids, the main effective compounds of LF, have a clear anti-inflammatory effect. The aim of this study was to clarify the mechanism of triterpenoids from LF on the treatment of RA.

\section{Methods}

A comprehensive network pharmacology strategy that consists of three sequential modules (pharmacophore matching, enrichment analysis and molecular docking) was carried out.

\section{Results}

We collected 21 triterpenoids from LF and predicted 61 targets strongly correlated with 15 biological processes and 8 related pathways. SYK and MAPK14 were key targets related to triterpenoids from LF and RA. LCK and GSK3B were potential targets and two effective triterpenoids from LF played a crucial role in the process of RA by targeting LCK and GSK3B.

\section{Conclusions}

The comprehensive strategy can serve as a universal method to illuminate the mechanism of triterpenoids from LF acting on treatment of RA by identifying the targets and pathways, and provided new ideas for RA treatment and evidence for clinical research and application of LF.

\section{Background}

Rheumatoid arthritis (RA) is a chronic inflammatory autoimmune disease that is affected by both genetic and environmental factors. The application of anti-rheumatic drugs and biological agents can improve the prognosis. However, there are still some patients who do not respond to current treatments, and biotherapeutics increases the risk of serious infections, which greatly limits the scope of clinical applications. Therefore, there is an urgent need to develop new therapeutic targets for RA therapy[1]. In the past few years, special attention has been paid to new genes and the close interaction between genetic factors and epigenetic mechanisms. The study also focused on the impact of environmental factors on disease development and new mechanisms that can affect the innate and adaptive immune systems at different stages of RA. However, many aspects of the disease require further study. Some 
exploration of different aspects of the pathogenesis of RA will help improve current diagnostic tools and help develop new targets for disease-changing therapies.

Liquidambaris Fructus (LF), a traditional Chinese medicine (TCM), is used for joint pain, numbness and edema[2]. It has small side effects and low price with great advantages and development potential[3]. Betulinic acid, a lupane-type triterpenoid, as one of the main compounds in LF, showed good antiinflammatory and analgesic effect[4]. According to the results of our preliminary research, LF showed good inhibitory effect on RA rats, suggesting that LF may be used as a potential anti-RA drug. However, whether triterpenoids in LF are effective compounds against RA needs to be further explored.

The compounds and mechanisms of TCM are numerous and complex. It is relatively difficult to detect the accurate mechanisms of TCM solely by conventional experimental method[5] because it realizes its particular therapeutic efficacy by modulating the biological network of body systems[6]. Consequently, the new and appropriate approaches are urgently needed to systematically and comprehensively dissect the mechanisms of TCM[7]. Owing to the rapid advancement of bioinformatics, the network pharmacology has become an emerging approach to efficiently and systemically disclose the molecular and pharmacological mechanisms of TCM $[8,9]$. Thus, we used the network pharmacology approach to study the possible effects and targets of triterpenoids from LF on the treatment of RA, which may provide new ideas for the RA therapy and the clinical application of LF.

\section{Methods}

\section{Data preparation and construction}

To collect the triterpenoids of LF, we used the Traditional Chinese Medicine Integrated Database (TCMID, http://www.megabionet.org/tcmid/), the TCM Database@Taiwan (http://tcm.cmu.edu.tw/), the Traditional Chinese Medicine Systems Pharmacology Database (TCMSP, http://lsp.nwu.edu.cn/) and some literature reports[10-12], 21 compounds were retrieved after deleting the duplicate data and saved as mol2 format (Table 1). We input all the active ingredients into PubChem (https://pubchem.ncbi.nlm.nih.gov/) and TCM Database@Taiwan and got the 3D molecular structure files of all triterpenoids from LF[13]. We imported these 3D molecular structure files into PharmMapper (http://lilab.ecust.edu.cn/pharmmapper/)[14]. The compounds without relevant 3D molecular structures information were removed. The top 99 targets of each compound acquired from PharmMapper were selected as potential targets in the present study. Thus, we collected 210 targets related to triterpenoids from LF after discarding duplicate data. The different genes associated with RA were gathered from Therapeutic Target Database (TTD) (http://bidd.nus.edu.sg/group/cjttd/)[15]. We searched the platform with keywords "Rheumatoid Arthritis" and selected 94 genes. The data of protein-protein interaction (PPI) came from String (https://string-db.org/) with the species limited to "Homo Sapiens"[16]. 
Table 1

21 effective compounds of triterpenoids of LF

\begin{tabular}{|c|c|c|}
\hline No. & Compound Name & Triterpenoids Type \\
\hline 1 & 2a,3ß-dihydroxy-23-norolean-4(24),12(13)-dien-28-oic acid & Oleanane-Type \\
\hline 2 & 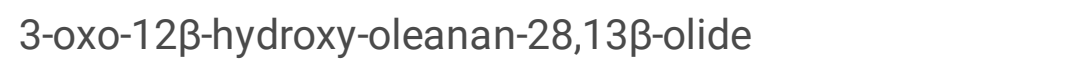 & Oleanane-Type \\
\hline 3 & 25-acetoxy-3a-hydroxyolean-12-en-28-oic acid & Oleanane-Type \\
\hline 4 & 11a-methoxyl-28-nor- $\beta$-amyrenone & Oleanane-Type \\
\hline 5 & 28-nor- $\beta$-amyrenone & Oleanane-Type \\
\hline 6 & Erythrodiol & Oleanane-Type \\
\hline 7 & Hydroxyoleanonic lactone & Oleanane-Type \\
\hline 8 & Lantanolic acid & Oleanane-Type \\
\hline 9 & Liquidambaric lactone & Oleanane-Type \\
\hline 10 & Oleanolic acid & Oleanane-Type \\
\hline 11 & Ambronal & Oleanane-Type \\
\hline 12 & Oleanonic acid & Oleanane-Type \\
\hline 13 & Arjunolic acid & Oleanane-Type \\
\hline 14 & a-amyrin & Oleanane-Type \\
\hline 15 & 6ß-hydroxy-3-oxo-lup-20(29)-en-28-oic acid & Lupane-Type \\
\hline 16 & Betulin & Lupane-Type \\
\hline 17 & Betulinic acid & Lupane-Type \\
\hline 18 & Betulonic acid & Lupane-Type \\
\hline 19 & Epibetulinic acid & Lupane-Type \\
\hline 20 & Ursolic acid & Ursane-Type \\
\hline 21 & Ursonic acid & Ursane-Type \\
\hline
\end{tabular}

\section{Network Construction And Enrichment Analysis}

The network visualization software Cytoscape (http://cytoscape.org/) was adopted to present all networks[17]. For each node in the interaction network, three topological features of each node in the network were calculated to find the major nodes. "Degree" is defined as the number of edges to node i; "Node betweenness" represents the number of shortest paths between pairs of nodes that run through node i; "Closeness" is the inverse of the sum of the distance from node i to other nodes. Then, 86 nodes 
with an average value of degree $\geq 14$, node betweenness $\geq 0.00103601$, and closeness $\geq 0.44097222$ were selected as major nodes.

Network construction was performed as follows: (1) compound-compound target network was built by 21 triterpenoids from LF and 30 corresponding targets; (2) compound target-RA targets' PPI network was established by linking 33 RA-related targets retrieved from TTD and 30 compound-related targets; (3) compound-compound target-RA targets' PPI network was constructed by compounds, compound-related targets and RA-related targets. Gene Ontology and Pathway Enrichment were performed through the database for Annotation, Visualization and Integrated Discovery (Metascape, http://metascape.org/gp/index.html)[18].

\section{Molecular Docking}

Molecular docking is a powerful computational tool which can predict the interaction energy between receptor and ligand. It determined the orientation of a ligand which would form the lowest energy complex within the receptor's binding pocket[19]. In this docking assay, 28 human receptors were retrieved from Protein Data Bank (PDB) with PDB ID: MAPK8 (3ELJ), MET (3ZCL), PGR (1SQN), ALB (5YOQ), PAR1 (5WS1), CDK2 (2R3R), BMP2 (4UI2), GSK3B (106L), MDM2 (5C5A), SYK (3FQS), MAPK14 (3FLN), AR (6D67), IGF1R (2OJ9), CASP3 (2DKO), MAPK1 (4ZZN), ESR1 (5DXE), KDR (2XIR), HPGDS (4EC0), PIK3CG (6AUD), HSP90AA1 (2YK9), REN (5UOF), PPARG (3V9V), CALM2 (5J03), NOS3 (4D1P), SRC (1043), EGFR (3W33), LCK (2IIM), F2 (5IUQ), while the 3D structure of SOD2 and ANXA5 were not available. During docking process, we used a rigid docking method, energy minimization of them in MOE2018, then we got 5 different conformations and other parameters were of default value.

\section{Results}

\section{Network construction and enrichment analysis}

We not only obtain an approximate observation of the relationship between bioactive compounds and compound targets but also discover the potential pharmacological effects of triterpenoids from LF from this network. These genes were likely to be the key or central genes in the development of RA. Finally, according to network stability, some targets are screened out, the entire network was split into two subnetworks with 47 targets and 18 targets, 61 nodes ( 28 compound-related target nodes, 31 RA-related target nodes and 2 common target nodes) and 656 edges remained (Fig. 1). Among them, 31 diseaserelated targets and 2 common targets for compounds and RA, are clear targets that are known to be related to RA, and 28 compound-related targets may be potential targets for triterpenoids from LF treatment of RA, so they are the objects of focus.

To clarify the multiple mechanisms of triterpenoids from LF on RA from a systematic level, we performed a GO enrichment analysis for the biological process, molecular function, and cellular compound of the 61 selected targets (Fig 2). The results suggested that the targets of LF were strongly correlated with 15 
biological processes (Table 2) and 8 related pathways (Table 3 ). This demonstrated that triterpenoids from LF probably worked by engaging in above biological processes, molecular functions, and cellular compound. Thus, our findings showed that triterpenoids from LF integrated multiple signaling pathways to modulate RA, endocrine system, immune system, signal transduction, cellular community, and cell motility. We found that triterpenoids from LF treatment of RA was not only related to the mechanism of action of traditional treatment of RA, but also showed many new pathways and biological processes.

Table 2 The representative gene ontology term with lowest lgP of each group 


G0:0043491: protein
kinase B signaling
G0:0050727:
regulation of
inflammatory response
GO:0033674: positive
regulation of kinase
activity

ingredient

disease

value

PIK3CG, KDR, SRC, PPARG,

CCR5, CD80, TNFSF11, CD86,

$-20.14$

HSP90AA1, CALM2, ESR1,

CD40, CD19, RELA, CCL2,

EGFR, LCK, IGF1R, MAPK1

CD40LG, IL1B, IL18

PIK3CG, KDR, SRC, PPARG, F2,

TNFRSF1A, TNFSF11, MMP9, $\quad-17.5$

ESR1, EGFR

IL17A, CD19, PTGS2, RELA, IL2,

CCR2, IL1B, IL10, IL18

PIK3CG, KDR, SRC, AR,

TNFSF11, IFNG, CD40, CD19,

REN, CASP3, HSP90AA1, F2, PTGS2, CCL2, IL2, CD40LG, IL1B,

CALM2, ESR1, EGFR,

IL10, PLA2G1B, IL18

IGF1R, ANXA5, MAPK1

GO:1903530:

SRC, REN, HSP90AA1,

TNFRSF1A, TNFSF11, IL1A, IL17A, -14.35

regulation of secretion

EGFR, ANXA5, MAPK1

IFNG, CD40, PTGS2, CCL2, IL2,

CD40LG, CASP1, CCR2, IL1B, IL10,

PLA2G1B

GO:0072507: divalent

PIK3CG, PPARG, F2, CALM2,

CCR5, TNFSF11, IFNG, CD40,

inorganic cation

homeostasis

NOS3, ESR1, EGFR, LCK,

CD19, PTGS2, CCL2, IL2, CCR2,

ANXA5, MAPK1

GO:0034614: cellular

SYK, MAPK14, CDK2,

CXCR3, IL1B, IL10, PLA2G1B

response to reactive

MAPK8, SOD2, BMP2,

SYK, MAPK14, JAK1, IL6, CSF1R,

TNF, JUN

oxygen species

GO:0071407: cellular

PARP1, ALB, MET, MDM2

PIK3CG, KDR, SRC, AR,

MMP9, IL1A, PTGS2, RELA,

response to organic

cyclic compound

REN, CASP3, PPARG,

NR3C1, CCL2, IL2, CSF2, CCR2,

CALM2, NOS3, ESR1, EGFR,

IL1B, IL10, IL18

\section{ANXA5, MAPK1}

GO:0051091: positive

PIK3CG, AR, REN, PPARG,

TNFRSF1A, TNFSF11, IL1A, IL17A, -12.09

regulation of DNA-

HPGDS, F2, ESR1, MAPK1

IFNG, CD40, CD19, PTGS2, RELA,

binding transcription

factor activity

GO:0050900: leukocyte

migration

PIK3CG, KDR, SRC, F2,

CCL2, CD40LG, CCR2, IL1B, IL10,

PLA2G1B, IL18

CCR5, TNFSF11, IL17A, RELA,

CALM2, NOS3, EGFR, LCK,

CCL2, CCR2, CXCR3, IL1B, IL10,

MAPK1

PLA2G1B

GO:0043065: positive

SRC, CASP3, PPARG, LCK,

TNFRSF1A, MMP9, IFNG, CD40, 


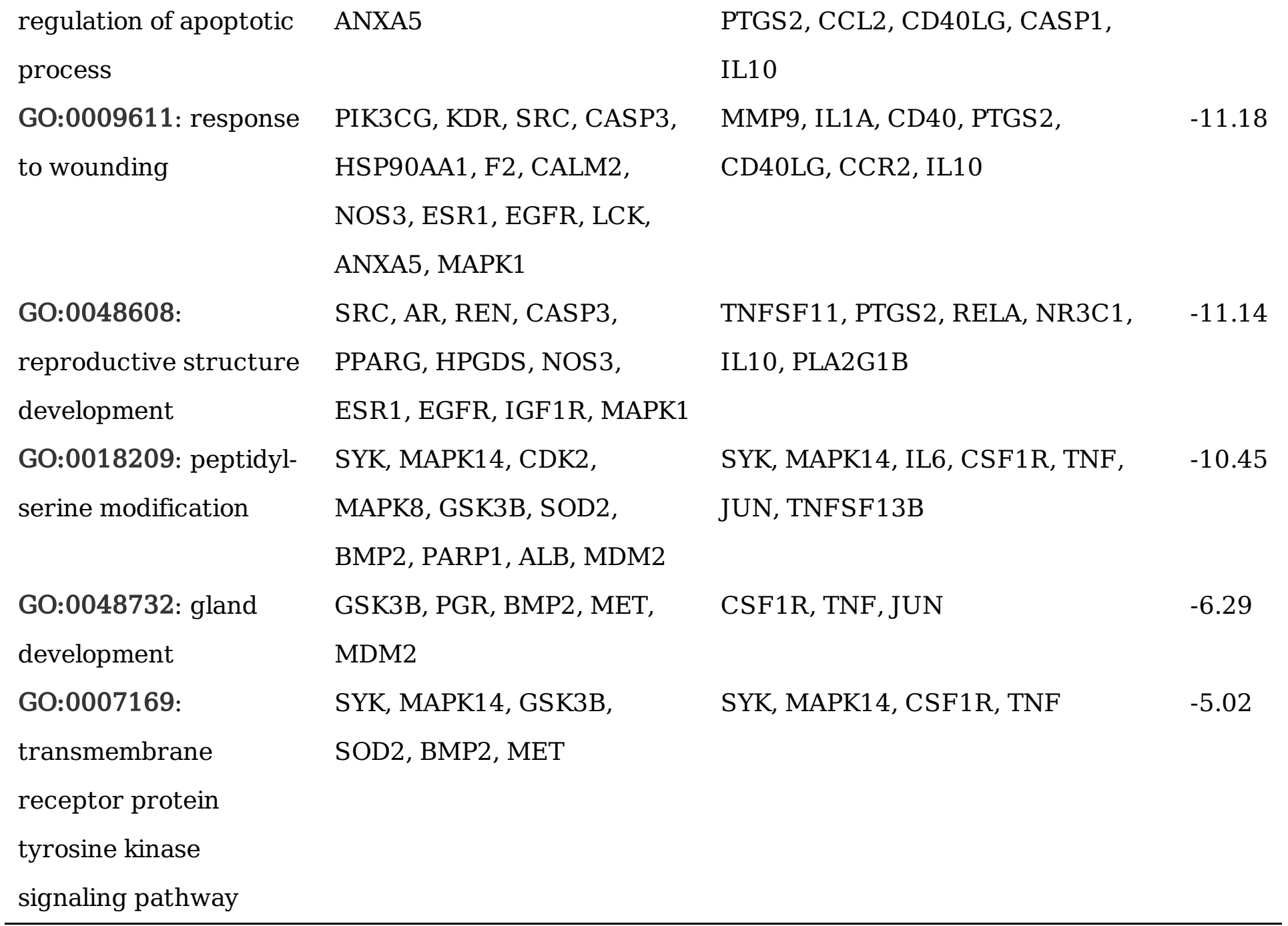

Table 3 The KEGG pathway in enrichment network 


\begin{tabular}{|c|c|c|c|}
\hline \multirow[t]{2}{*}{ KEGG Pathway } & \multicolumn{2}{|c|}{ Target Gene } & \multirow{2}{*}{$\begin{array}{l}\text { p- } \\
\text { value }\end{array}$} \\
\hline & ingredient & disease & \\
\hline hsa04657: & PIK3CG, SRC, CASP3, & TNFRSF1A, TNFSF11, MMP9, IL1A, & -17.11 \\
\hline \multirow[t]{3}{*}{ IL-17 signaling pathway } & PPARG, HSP90AA1, & IL17A, IFNG, PTGS2, RELA, CCL2, & \\
\hline & CALM2, NOS3, EGFR, LCK, & CSF2, CASP1, IL1B, IL10, IL18 & \\
\hline & MAPK1 & & \\
\hline hsa05418: & KDR, SRC, AR, CASP3, & TNFRSF1A, MMP9, IL1A, IFNG, & -16.86 \\
\hline Fluid shear stress and & HSP90AA1, CALM2, NOS3, & CD19, PTGS2, RELA, CCL2, IL2, & \\
\hline atherosclerosis & LCK, MAPK1 & CSF2, CASP1, IL1B & \\
\hline \multirow[t]{3}{*}{ hsa05145: Toxoplasmosis } & PIK3CG, KDR, SRC, CASP3, & TNFRSF1A, CCR5, CD80, IFNG, & -14.25 \\
\hline & LCK, MAPK1 & CD86, CD40, CD19, RELA, CCL2, & \\
\hline & & IL2, CD40LG, IL1B, IL10, IL18 & \\
\hline hsa05200: Pathways in & SYK, MAPK14, CDK2, & SYK, MAPK14, JAK1, IL6, CSF1R, & -13.32 \\
\hline \multirow[t]{2}{*}{ cancer } & MAPK8, GSK3B, SOD2, & TNF, JUN & \\
\hline & BMP2, MET, MDM2 & & \\
\hline hsa04380: Osteoclast & SYK, MAPK14, CDK2, & SYK, MAPK14, JAK1, IL6, CSF1R, & -12.22 \\
\hline \multirow[t]{2}{*}{ differentiation } & MAPK8, GSK3B, SOD2, & TNF, JUN, TNFSF13B & \\
\hline & BMP2, PARP1, MDM2 & & \\
\hline hsa05169: Epstein-Barr & SYK, MAPK14, CDK2, & SYK, MAPK14, JAK1, IL6, JUN & -12.09 \\
\hline \multirow[t]{2}{*}{ virus infection } & MAPK8, GSK3B, SOD2, & & \\
\hline & MET, MDM2 & & \\
\hline \multirow[t]{3}{*}{ hsa05152: Tuberculosis } & SYK, MAPK14, GSK3B, & SYK, MAPK14, JAK1, IL6, CSF1R, & -8.93 \\
\hline & BMP2, PARP1, ALB, MET, & TNF, JUN, TNFSF13B & \\
\hline & MDM2 & & \\
\hline \multirow[t]{2}{*}{ hsa05160: Hepatitis C } & MAPK14, MAPK8, GSK3B, & MAPK14, JAK1, IL6, TNF & -7.45 \\
\hline & PGR, PARP1 & & \\
\hline
\end{tabular}

\section{Molecular Docking}

We docked 28 compound-related targets with 21 compounds and screened 23 targets according to the docking effect. According to the principle of minimum energy and maximum similarity of ligand-receptor binding sites, we selected 2 best performing targets (LCK and GSK3B) from the docking results. Tyrosine kinases LCK is involved in inflammatory disorders. The potent and selective LCK inhibitors exhibited excellent anti-inflammatory effect and for the potential application in inflammatory disorders including 
RA[20]. Re-organisation of the macrophage metabolism in patients with RA drives unopposed oxygen consumption and ultimately, excessive production of tissue-destructive enzymes. The underlying molecular defect relates to the deactivation of GSK3B, which controls mitochondrial fuel influx and as such represents a potential therapeutic target for anti-inflammatory therapy[21]. Although they are not clear targets related to RA, they may be important potential targets. The best compounds to dock with

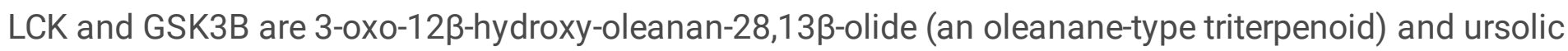
acid (an ursane-type triterpenoid), and their binding sites are shown in the Fig. 3 and Table 4.

Table 4

Key amino acids in active pocket around ligand after docking

\begin{tabular}{|lll|}
\hline Ligand & 2iim (LCK) & 106I (GSK3B) \\
\hline Built-in ligand & $\begin{array}{l}\text { Asp77 His76 Asp79 Trp97 } \\
\text { Phe110 }\end{array}$ \\
\hline $\begin{array}{l}\text { Compound 2 } \\
\text { (docking) }\end{array}$ & His76 Asp79 Phe110 Trp97 \\
\hline $\begin{array}{l}\text { Built-in ligand } \\
\begin{array}{l}\text { Compound 20 } \\
\text { (docking) }\end{array}\end{array}$ & $\begin{array}{l}\text { Glu236 Glu279 Lys277 Ser9 Lys181 Asn280 } \\
\text { Asp293 }\end{array}$ \\
\hline
\end{tabular}

\section{Discussion}

We discovered that LF, as a TCM, significantly alleviated the symptoms of RA and believe that its impact may be related to its potential function. Therefore, we explored the potential mechanism by the network pharmacology approach. RA is a chronic inflammatory autoimmune disease. The basic pathological changes of RA include infiltration of inflammatory cells, hyperplasia of synovium, pannus formation and resulting cartilage and bone damage.

The hyperplastic growth of RA fibroblast-like synoviocytes (FLSs) and inflammatory response are pathological hallmarks of RA. Downregulation of AKT2 significantly prevents RA-FLS cell proliferation and migration[22]. The IL-17 signaling pathway can mediate the proliferation and invasion of HFLS-RA, thereby regulating the expression of MMP13 in HFLS-RA and thereby participating in the degradation of chondrocytes[23]. By inhibiting the activation of MAPK-ERK1 / 2 signaling pathway, FLS proliferation can be reduced and apoptosis can be promoted[24]. Osteoclasts are the cells responsible for bone erosion in RA synovial joints. Th17 cells induce the production of RANKL by FLS which promotes osteoclastogenesis[25]. Cytokines mainly TNF-a, IL-1 $\beta$, and IL-6 amplify osteoclastogenesis[26]. Under the inflammatory environment of RA, activated macrophages revealed increased expression of folate receptor and elevated intracellular reactive oxygen species (ROS) level[27]. Bruton's tyrosine kinase (BTK) regulates critical signal transduction pathways involved in the pathobiology of rheumatoid arthritis (RA) and other autoimmune disorders[28]. In addition, we also discovered some new possible pathways, such 
as pathways in cancer and reproductive structure development, which might provide new ideas for research on RA treatment.

Although the docking results of SYK and MAPK14 are not the best, they are common targets for compounds and RA. RA is is an inflammatory autoimmune disease in which autoantibodies, immune complexes, and autoreactive T cells account for the expression of tissue injury and inflammation. Spleen tyrosine kinase (SYK) is a member of the Src family of non-receptor tyrosine kinases, which associates directly with surface receptors, including B-cell receptor and Fcy receptor, and is involved in a variety of signal transduction pathways. SYK inhibitors efficiently eliminate these alterations[29]. p38 Mitogenactivated protein kinases (MAPK) are key regulators of cell differentiation and apoptosis through different signaling cascades. There are four isoforms of p38 MAPK: p38a (MAPK14), p38ß (MAPK11), p38y (MAPK12 or ERK6) and p38ס (MAPK13 or SAPK4). p38 MAPK associate with many pathogenic processes in RA, including production of proinflammatory cytokines and matrix metalloproteinase (MMPs). Several MAPKs are activated at different sites in RA synovium; 14 p38a activation are found in synovial lining and endothelial cells, which play a key role in TNF-mediated inflammatory bone destruction in RA. p38 MAPK inhibitors reduce the production of cytokines and MMP in rheumatoid synovial cells[30].

In addition to SYK and MAPK14, MAPK1 and PIK3CG are arthritis-related targets, ESR1 and IGF1R are related to multiple bone disease and joint pain, AR and PGR are osteoporosis-related targets, and SRC, CASP3, CDK2, EGFR and MET are cancer Related targets, NOS and CALM2 are targets for heart-related diseases, while PPARG is associated with diabetes. Therefore, the role of targets of other diseases in RA needs our attention and further research.

However, there are some limitations in using network pharmacology methods to predict active ingredients and potential mechanisms. (i) The active ingredients screened may be inconsistent with the ingredients actually absorbed in the blood of RA patients; (ii) it is difficult to distinguish between inhibition and activation; (iii) the prediction results may be affected by possible deviations in highly researched path methods and functions and (iv) the interaction between nodes in the network construction methods are still unclear. Therefore, the specific actions and properties need to be further verified and probed in future studies.

\section{Conclusions}

We collected 21 triterpenoids from LF and predicted 61 potential targets (28 compound-related targets, 31 RA-related targets and 2 common targets for compounds and RA). The network analysis uncovered that triterpenoids from LF probably exerted its pharmacological effects on RA by modulating certain targets, such as SYK and MAPK14, and some potential targets like LCK and GSK3B according to the docking effect. The $\mathrm{GO}$ analysis of targets disclosed that the Triterpenoids from LF possibly produced synergistic effects for treating RA mainly by regulating protein kinase B signaling, regulation of inflammatory response and other biological processes. Meanwhile, the pathway analysis in our work indicated that 
triterpenoids from LF might simultaneously act on IL-17 signaling pathway, fluid shear stress and atherosclerosis and other signaling pathways associated with the pathogenesis of RA. In summary, the current study combines active ingredients, target prediction, network analysis, GO enrichment analysis, pathway analysis and molecular docking by a network pharmacology method to illuminate the molecular and pharmacological mechanism of triterpenoids of triterpenoids from LF against RA from a systematic perspective. Meanwhile, based on the systematic analysis for the bioactive compounds, crucial targets, and key pathways of triterpenoids from LF against RA, our research unveiled that triterpenoids from LF is characterized by multi-compound and multi-target synergistic treatment. New potential targets and pathways for triterpenoids from LF on the treatment of RA were discovered in the study, which provided new ideas for RA treatment and provided evidence for clinical research and application of LF.

\section{Abbreviations}

RA: Rheumatoid arthritis; LF: Liquidambaris Fructus; TCM: traditional Chinese medicine; TCMID: Traditional Chinese Medicine Integrated Database; TCMSP: Traditional Chinese Medicine Systems Pharmacology Database; TTD: Therapeutic Target Database; PPI: protein-protein interaction; PDB: Protein Data Bank; FLSs: fibroblast-like synoviocytes; ROS: reactive oxygen species; BTK: Bruton's tyrosine kinase; SYK: Spleen tyrosine kinase; MAPK: Mitogen-activated protein kinases; MET: Hepatocyte growth factor receptor; PGR: Progesterone receptor; ALB: Serum albumin; PAR1: Proteinase-activated receptor 1; CDK2: Cyclin-dependent kinase 2; BMP2: Bone morphogenetic protein 2; GSK3B: Glycogen synthase kinase-3 beta; MDM2: E3 ubiquitin-protein ligase Mdm2; AR: Androgen receptor; IGF1R: Insulin-like growth factor 1 receptor; CASP3: Caspase-3, ESR1: Estrogen receptor; KDR: Vascular endothelial growth factor receptor 2; HPGDS: Hematopoietic prostaglandin D synthase; PIK3CG: Phosphatidylinositol 4,5bisphosphate 3-kinase catalytic subunit gamma isoform; HSP90AA1: Heat shock protein HSP 90-alpha; REN: Renin; PPARG: Peroxisome proliferator-activated receptor gamma; CALM2: Calmodulin-2; NOS3: Nitric oxide synthase, endothelial; SRC: Proto-oncogene tyrosine-protein kinase Src; EGFR: Epidermal growth factor receptor; LCK: Tyrosine-protein kinase Lck; F2: Prothrombin; SOD2: Superoxide dismutase [Mn], mitochondrial; ANXA5: Annexin A5; MMPs: matrix metalloproteinase; GO: Gene Ontology; KEGG: Kyoto Encyclopedia of Genes and Genomes.

\section{Declarations}

\section{Ethics approval and consent to participate}

Not applicable.

\section{Consent for publication}

Not applicable.

\section{Availability of data and materials}


The datasets used and/or analyzed during the current study are available from the corresponding author on reasonable request.

\section{Competing interests}

The authors declare that they have no competing interests.

\section{Funding}

This work was supported by the Natural Science Foundation of Liaoning Province (Grant No.20170541028).

\section{Authors' contributions}

Data curation, $\mathrm{W}-\mathrm{XL}$ and $\mathrm{PQ}$; Formal analysis, $\mathrm{W}-\mathrm{XL}$ and $\mathrm{PQ}$; Methodology, $\mathrm{W}-\mathrm{XL}$ and $\mathrm{PQ}$; Resources, LG and D-FZ; Supervision, PQ; Writing-original draft, W-XL; Writing-review and editing, PQ and D-FZ. All authors read and approved the final manuscript.

\section{Acknowledgements}

Not applicable.

\section{References}

1. Croia C, Bursi R, Sutera D, Petrelli F, Alunno A, Puxeddu I. One year in review 2019: pathogenesis of rheumatoid arthritis. Clin Exp Rheumatol [Internet]. 2019/05/10. Italy; 2019;37:347-57. Available from: https://pubmed.ncbi.nlm.nih.gov/31111823.

2. National Pharmacopoeia Committee. Pharmacopoeia of People's Republic of China [M ]. Part 1. Beijing.: Chemical Industry Press.; 2015. p. 356-357.

3. Moudgil KD, Berman BM. Traditional Chinese medicine: potential for clinical treatment of rheumatoid arthritis. Expert Rev Clin Immunol [Internet]. 2014/05/12. England; 2014;10:819-22. Available from: https://pubmed.ncbi.nlm.nih.gov/24820012.

4. Ou Z, Zhao J, Zhu L, Huang L, Ma Y, Ma C, et al. Anti-inflammatory effect and potential mechanism of betulinic acid on $\lambda$-carrageenan-induced paw edema in mice. Biomed Pharmacother [Internet]. 2019/08/16. France; 2019;118:109347. Available from: https://pubmed.ncbi.nlm.nih.gov/31545273.

5. Wang F, Cheng X-L, Li Y-J, Shi S, Liu J-K ent-Pimarane diterpenoids from Siegesbeckia orientalis and structure revision of a related compound. J Nat Prod [Internet]. United States; 2009;72:2005-8. Available from: https://pubmed.ncbi.nlm.nih.gov/19813758.

6. Kim Y-S, Kim H, Jung E, Kim J-H, Hwang W, Kang E-J, et al. A novel antibacterial compound from Siegesbeckia glabrescens. Molecules [Internet]. MDPI; 2012;17:12469-77. Available from: https://pubmed.ncbi.nlm.nih.gov/23095892. 
7. Li H, Kim JY, Hyeon J, Lee HJ, Ryu J-H. In vitro antiinflammatory activity of a new sesquiterpene lactone isolated from Siegesbeckia glabrescens. Phytother Res [Internet]. 2011/02/10. England; 2011;25:1323-7. Available from: https://pubmed.ncbi.nlm.nih.gov/21308823.

8. Wang R, Liu Y-Q, Ha W, Shi Y-P, Hwang T-L, Huang G-J, et al. In vitro anti-inflammatory effects of diterpenoids and sesquiterpenoids from traditional Chinese medicine Siegesbeckia pubescens. Bioorg Med Chem Lett [Internet]. 2014/06/19. England; 2014;24:3944-7. Available from: https://pubmed.ncbi.nlm.nih.gov/24997688.

9. Yamamoto A, Fukuda A, Seto H, Miyazaki T, Kadono Y, Sawada Y, et al. Suppression of arthritic bone destruction by adenovirus-mediated dominant-negative Ras gene transfer to synoviocytes and osteoclasts. Arthritis Rheum [Internet]. United States; 2003;48:2682-92. Available from: https://pubmed.ncbi.nlm.nih.gov/13130489.

10. Huang L, Xie D, Yu Y, Liu H, Shi Y, Shi T, et al. TCMID 2.0: a comprehensive resource for TCM. Nucleic Acids Res [Internet]. Oxford University Press; 2018;46:D1117-20. Available from: https://pubmed.ncbi.nlm.nih.gov/29106634.

11. Chen CY-C. TCM Database@Taiwan: the world's largest traditional Chinese medicine database for drug screening in silico. PLoS One [Internet]. Public Library of Science; 2011;6:e15939-e15939. Available from: https://pubmed.ncbi.nlm.nih.gov/21253603.

12. Ru J, Li P, Wang J, Zhou W, Li B, Huang C, et al. TCMSP: a database of systems pharmacology for drug discovery from herbal medicines. J Cheminform [Internet]. BioMed Central; 2014;6:13. Available from: https://pubmed.ncbi.nlm.nih.gov/24735618.

13. Kim S. Getting the most out of PubChem for virtual screening. Expert Opin Drug Discov [Internet]. 2016/08/05. 2016;11:843-55. Available from: https://pubmed.ncbi.nlm.nih.gov/27454129.

14. Wang X, Shen Y, Wang S, Li S, Zhang W, Liu X, et al. PharmMapper 2017 update: a web server for potential drug target identification with a comprehensive target pharmacophore database. Nucleic Acids Res [Internet]. Oxford University Press; 2017;45:W356-60. Available from: https://pubmed.ncbi.nlm.nih.gov/28472422.

15. Chen X, Ji ZL, Chen YZ. TTD: Therapeutic Target Database. Nucleic Acids Res [Internet]. Oxford University Press; 2002;30:412-5. Available from: https://pubmed.ncbi.nlm.nih.gov/11752352.

16. Szklarczyk D, Morris JH, Cook H, Kuhn M, Wyder S, Simonovic M, et al. The STRING database in 2017: quality-controlled protein-protein association networks, made broadly accessible. Nucleic Acids Res [Internet]. 2016/10/18. Oxford University Press; 2017;45:D362-8. Available from: https://pubmed.ncbi.nlm.nih.gov/27924014.

17. Shannon P, Markiel A, Ozier O, Baliga NS, Wang JT, Ramage D, et al. Cytoscape: a software environment for integrated models of biomolecular interaction networks. Genome Res [Internet]. Cold Spring Harbor Laboratory Press; 2003;13:2498-504. Available from: https://pubmed.ncbi.nlm.nih.gov/14597658.

18. Zhou Y, Zhou B, Pache L, Chang M, Khodabakhshi AH, Tanaseichuk O, et al. Metascape provides a biologist-oriented resource for the analysis of systems-level datasets. Nat Commun [Internet]. Nature 
Publishing Group UK; 2019;10:1523. Available from: https://pubmed.ncbi.nlm.nih.gov/30944313.

19. Hsin K-Y, Ghosh S, Kitano H. Combining machine learning systems and multiple docking simulation packages to improve docking prediction reliability for network pharmacology. PLoS One [Internet]. Public Library of Science; 2013;8:e83922-e83922. Available from:

https://pubmed.ncbi.nlm.nih.gov/24391846.

20. Farag AK, Elkamhawy A, Londhe AM, Lee K-T, Pae AN, Roh EJ. Novel LCK/FMS inhibitors based on phenoxypyrimidine scaffold as potential treatment for inflammatory disorders. Eur J Med Chem [Internet]. 2017/10/04. France; 2017;141:657-75. Available from:

https://pubmed.ncbi.nlm.nih.gov/29107425.

21. Zeisbrich M, Yanes RE, Zhang H, Watanabe R, Li Y, Brosig L, et al. Hypermetabolic macrophages in rheumatoid arthritis and coronary artery disease due to glycogen synthase kinase $3 \mathrm{~b}$ inactivation. Ann Rheum Dis [Internet]. 2018/02/03. 2018;77:1053-62. Available from: https://pubmed.ncbi.nlm.nih.gov/29431119.

22. Yu F-Y, Xie C-Q, Jiang C-L, Sun J-T, Feng H-C, Li C, et al. MiR-92a inhibits fibroblast-like synoviocyte proliferation and migration in rheumatoid arthritis by targeting AKT2. J Biosci [Internet]. India; 2018;43:911-9. Available from: https://www.ncbi.nlm.nih.gov/pubmed/30541951.

23. Su J, Zhang J, Zhu J, Liu Y. The promoting effect of MMP13 on mediating the development of HFLSRA by the target of miR-19a through IL-17 signaling pathway. [Internet]. J. Cell. Biochem. Department of Rheumatology and Immunology, West China Hospital, Sichuan University, Chengdu, China.; 2020. Available from: https://pubmed.ncbi.nlm.nih.gov/31960999.

24. Lin K, Su H-Y, Jiang L-F, Chu T-G, Li Z, Chen X-L, et al. Influences of miR-320a on proliferation and apoptosis of fibroblast-like synoviocytes in rheumatoid arthritis through targeting MAPK-ERK1/2. Eur Rev Med Pharmacol Sci [Internet]. Italy; 2019;23:1907-14. Available from: https://www.ncbi.nlm.nih.gov/pubmed/30915733.

25. Rana AK, Li Y, Dang Q, Yang F. Monocytes in rheumatoid arthritis: Circulating precursors of macrophages and osteoclasts and, their heterogeneity and plasticity role in RA pathogenesis. Int Immunopharmacol [Internet]. 2018/10/23. Netherlands; 2018;65:348-59. Available from: https://www.ncbi.nlm.nih.gov/pubmed/30366278.

26. Meng Q, Du X, Wang H, Gu H, Zhan J, Zhou Z. Astragalus polysaccharides inhibits cell growth and pro-inflammatory response in IL-1 $\beta$-stimulated fibroblast-like synoviocytes by enhancement of autophagy via PI3K/AKT/mTOR inhibition. Apoptosis [Internet]. Netherlands; 2017;22:1138-46. Available from: https://www.ncbi.nlm.nih.gov/pubmed/28660311.

27. Chen M, Amerigos JCKD, Su Z, Guissi NEl, Xiao Y, Zong L, et al. Folate Receptor-Targeting and Reactive Oxygen Species-Responsive Liposomal Formulation of Methotrexate for Treatment of Rheumatoid Arthritis. Pharmaceutics [Internet]. MDPl; 2019;11:582. Available from: https://www.ncbi.nlm.nih.gov/pubmed/31698794.

28. Gillooly KM, Pulicicchio C, Pattoli MA, Cheng L, Skala S, Heimrich EM, et al. Bruton's tyrosine kinase inhibitor BMS-986142 in experimental models of rheumatoid arthritis enhances efficacy of agents 
representing clinical standard-of-care. PLoS One [Internet]. Public Library of Science; 2017;12:e0181782-e0181782. Available from: https://www.ncbi.nlm.nih.gov/pubmed/28742141.

29. Deng GM, Kyttaris VC, Tsokos GC. Targeting syk in autoimmune rheumatic diseases. Front Immunol. 2016;7:1-5.

30. Coulthard LR, Taylor JC, Eyre S, Robinson JI, Wilson AG, Isaacs JD, et al. Genetic variants within the MAP kinase signaling network and anti-TNF treatment response in Rheumatoid arthritis patients. Ann Rheum Dis. 2011;70:98-103.

\section{Figures}




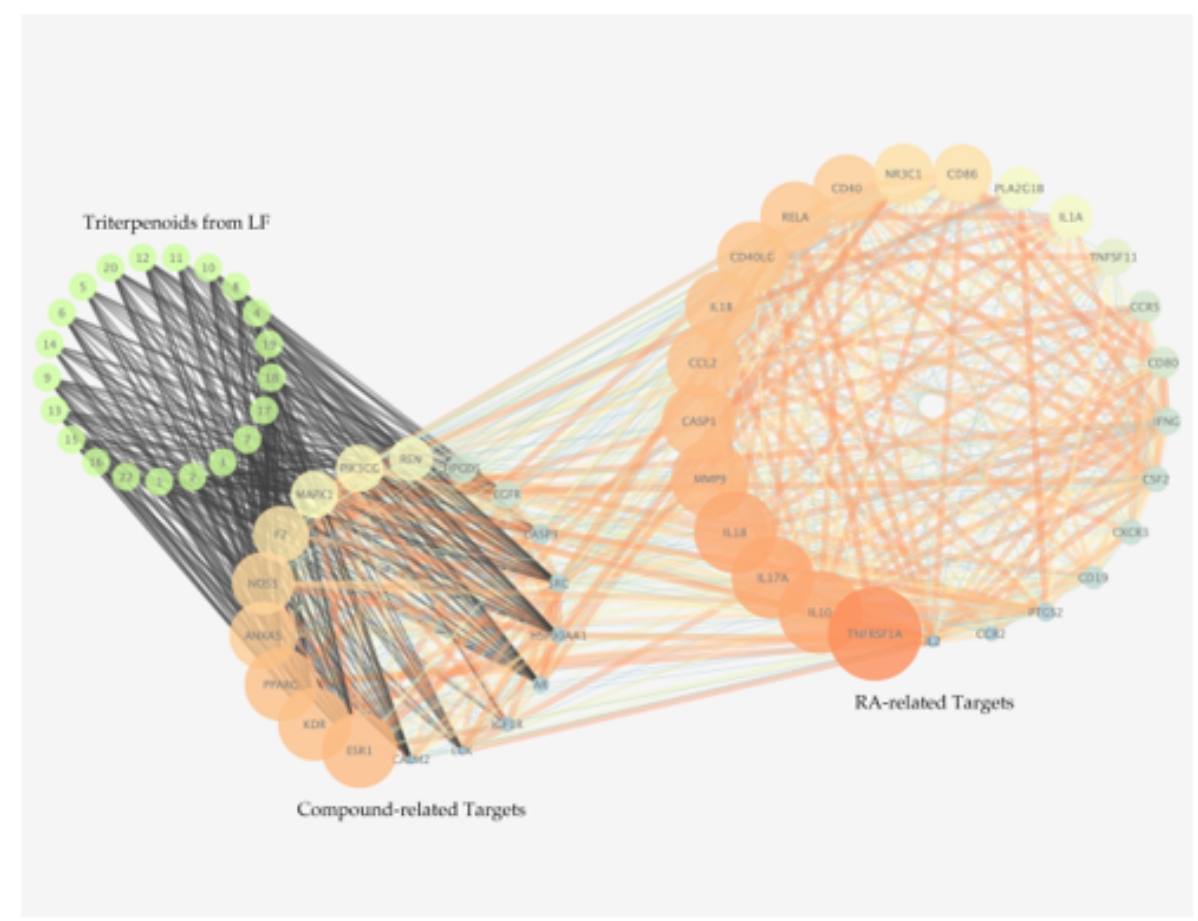

b

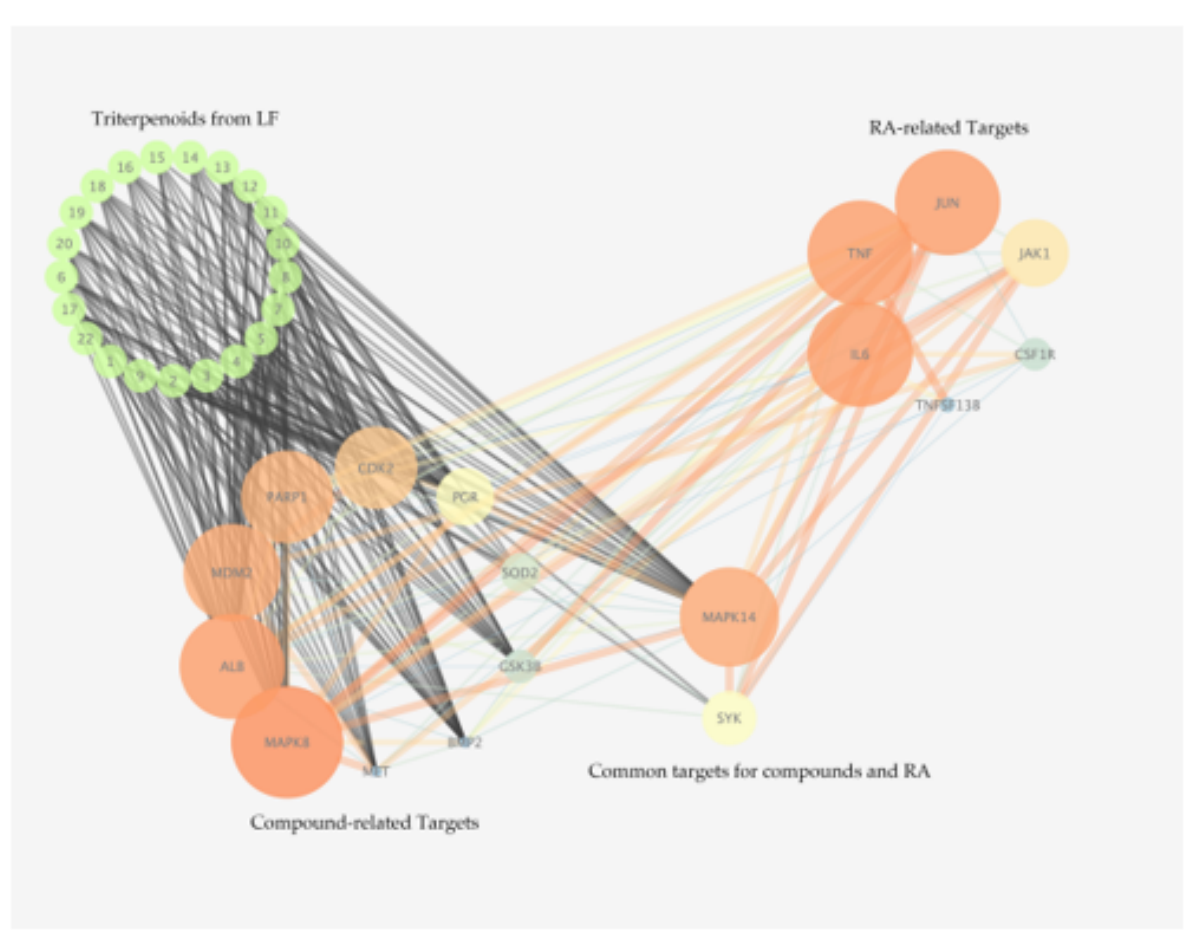

Figure 1

Compound-Compound Target-RA Targets' PPI Networks with 43 targets(a) and 18 targets(b) 


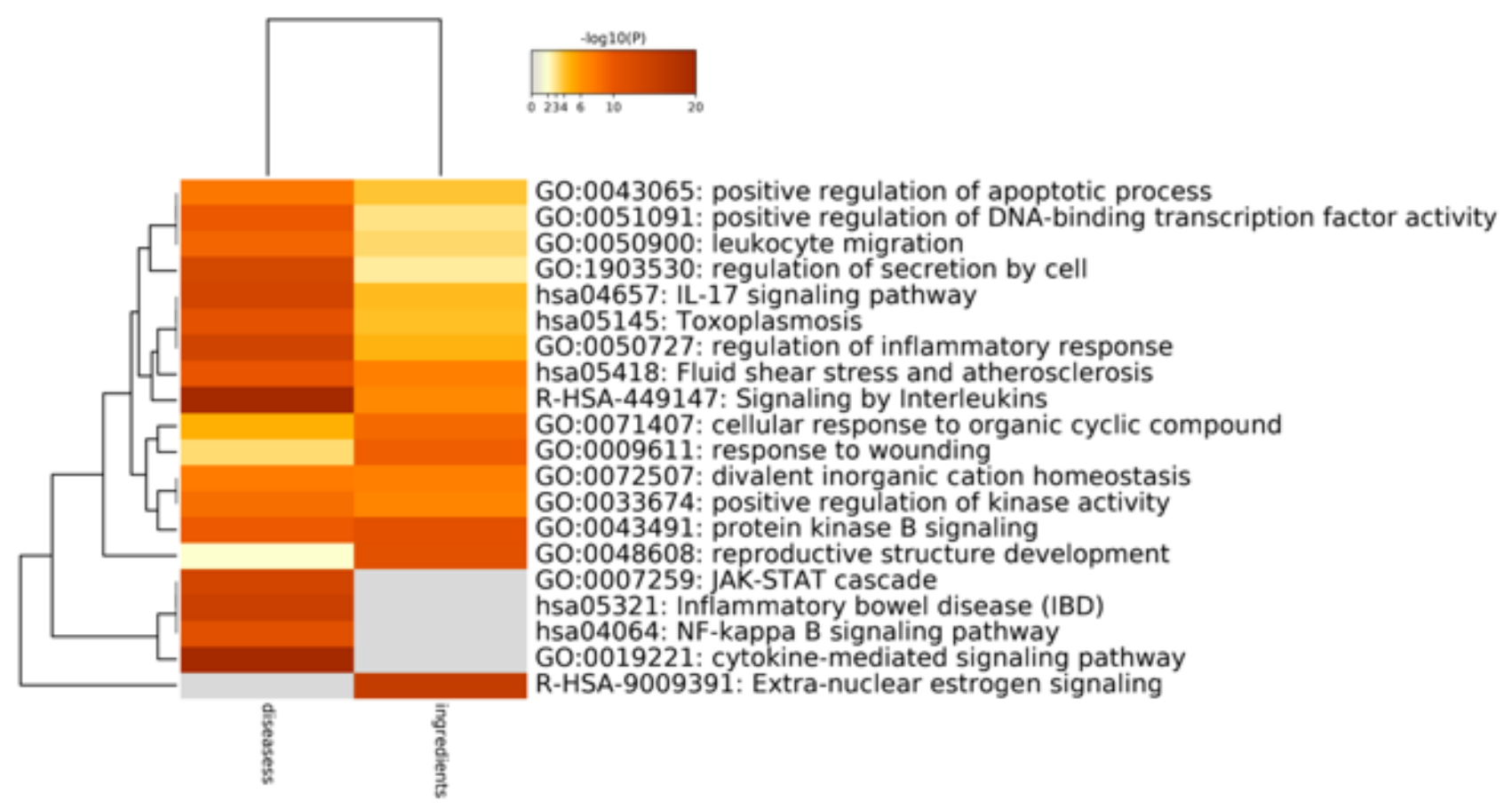

b

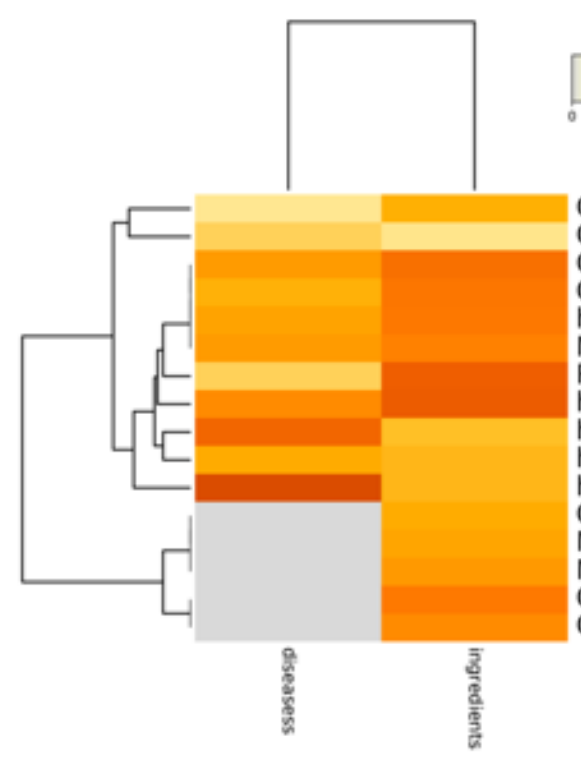

$-\log 1009$

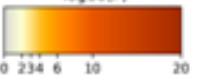

GO:0007169: transmembrane receptor protein tyrosine kinase signaling pathway GO:0048732: gland development

GO:0018209: peptidyl-serine modification

GO:0034614: cellular response to reactive oxygen species

hsa05200: Pathways in cancer

M115: PID REG GR PATHWAY

R-HSA-2262752: Cellular responses to stress

hsa05169: Epstein-Barr virus infection

hsa05152: Tuberculosis

hsa05160: Hepatitis C

hsa04380: Osteoclast differentiation

GO:1900407: regulation of cellular response to oxidative stress

M81: PID CDC42 PATHWAY

M136: PID FOXO PATHWAY

GO:0080135: regulation of cellular response to stress

GO:1903829: positive regulation of cellular protein localization

\section{Figure 2}

GO and Pathway Enrichment Analyses HeatmapSelectedGO with 47 targets(a) and $18 \operatorname{targets(b)}$ 
(쇼욤

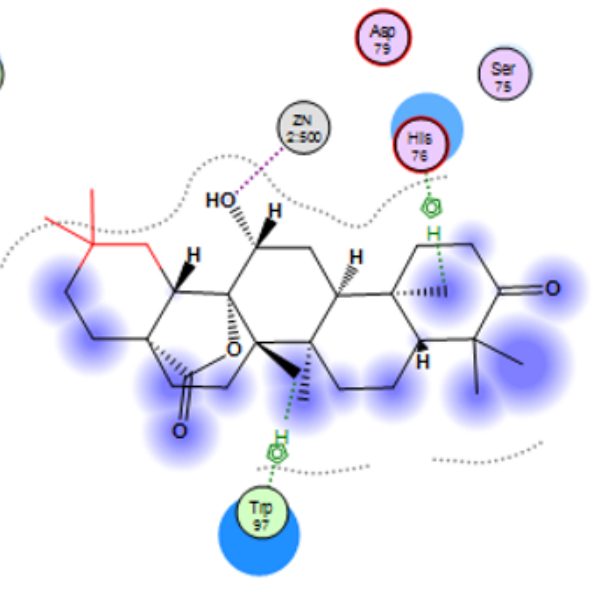

$\mathbf{a}$

b

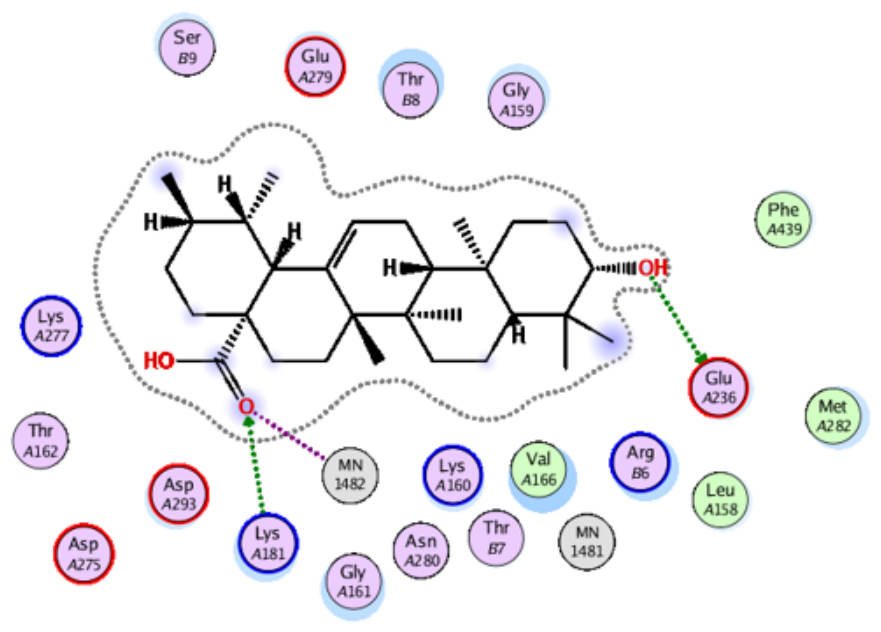

\section{Figure 3}

The dock results of compound2-2iim(a) and compound20-106I(b)

\section{Supplementary Files}

This is a list of supplementary files associated with this preprint. Click to download.

- Additionalfile2.xlsx

- Additionalfile2.xlsx

- Additionalfile1.xlsx

- Additionalfile1.xlsx 